\title{
What Makes Community Health Worker Models for Tuberculosis Active Case Finding Work? A Cross- sectional Study of TB REACH Projects to Identify Success Factors for Increasing Case Notifications
}

\section{Thu Dam}

Friends for International TB Relief

Rachel Forse ( $\square$ rachel.forse@tbhelp.org )

Friends for International Tuberculosis Relief https://orcid.org/0000-0002-0716-3342

Phuong Tran

Friends for International TB Relief

Luan Vo

Friends for International TB Relief \& IRD VN

Andrew James Codlin

Friends for International TB Relief

\section{Lan Nguyen}

Centre for Development of Community Health Initiatives

Jacob Creswell

Stop TB Partnership

\section{Research}

Keywords: Tuberculosis, community healthcare workers, TB REACH, Global Strategy, Workforce 2030

Posted Date: September 13th, 2021

DOl: https://doi.org/10.21203/rs.3.rs-850489/v1

License: (9) (i) This work is licensed under a Creative Commons Attribution 4.0 International License. Read Full License 


\section{Abstract}

Background: In the field of tuberculosis (TB), Community Healthcare Workers (CHWs) have been engaged for advocacy, case detection, and patient support in a wide range of settings. Estimates predict large-sale shortfalls of healthcare workers in low- and middle-income settings by 2030 and strategies are needed to optimize the health workforce to achieve universal availability and accessibility of healthcare. In 2018, the World Health Organization (WHO) published guidelines on best practices for $\mathrm{CHW}$ engagement, and identified remaining knowledge gaps. Stop TB Partnership's TB REACH initiative has supported interventions using $\mathrm{CHWs}$ to deliver TB care in over 30 countries, and utilized the same primary indicator to measure project impact at the population-level for all TB active case finding projects, which makes the results comparable across multiple settings. This study compiled 10 years of implementation data from the initiative's grantee network to begin to address key knowledge gaps in CHW networks.

Methods: We conducted a cross-sectional study analyzing the TB REACH data repository $(n=123)$ and primary survey responses $(n=50)$ of project implementers. We designed a survey based on WHO guidelines to understand projects' practices on CHW recruitment, training, activities, supervision, compensation, and sustainability. We segmented projects by TB notification impact and fitted linear random-effect regression models to identify practices associated with higher changes in notifications.

Results: Most projects employed CHWs for advocacy alongside case finding and holding activities. Model characteristics associated with higher project impact included incorporating e-learning in training and having the prospect of $\mathrm{CHWs}$ continuing their responsibilities at the close of a project. Factors that trended towards being associated with higher impact were community-based training, differentiated contracts, and non-monetary incentives.

Conclusion: In line with WHO guidelines, our findings emphasize that successful implementation approaches provide $\mathrm{CHWs}$ with comprehensive training, continuous supervision, fair compensation, and are integrated within the existing primary healthcare system. However, we encountered a great degree of heterogeneity in $\mathrm{CHW}$ engagement models, resulting in few practices clearly associated with higher notifications.

\section{Background}

Since the adoption of the Alma Ata Declaration in 1978 and most recently the Astana Declaration in 2018, primary care with community health workers (CHWs) has been considered a critical path to attain healthcare for all. 1,2 The specific definition of CHWs can differ, with the term encompassing a diverse group of "lay and educated, formal and informal, paid and unpaid health workers." These CHWs provide services such as health education and can refer or support individuals and families seeking preventative or curative care. In recognition of the importance of community networks in health, the Global Strategy on Human Resources for Health: Workforce 2030 in 2016 was adopted by the 69th World Health Assembly and subsequently the World Health Organization (WHO) issued guidelines on health policy and system 
support to optimize CHW programs. ${ }^{3,4}$ These guidelines specifically offered a set of recommendations on CHWs selection, training, management and supervision, career advancement, community embeddedness, and health system support.

Despite substantial evidence on CHWs and their positive impact on communities, ${ }^{5,6}$ there remain key knowledge gaps. In particular, the WHO guidelines cited knowledge gaps across six key themes: 1) intervention activities; 2) recruitment/selection; 3) training; 4) compensation/remuneration; 5) supervision; and 6) sustainable integration into the health system. ${ }^{4}$ Moreover, studies have decried the lack of evidence on how to integrate and support CHWs within health systems and the "rights and needs of CHWs". ${ }^{5}$ Another review posited that efficacy assessments of $\mathrm{CHW}$ projects were carried out under ideal circumstances, leading to a need for more evidence under "real world" conditions. ${ }^{7}$

The current COVID-19 pandemic notwithstanding, with 10 million cases and 1.4 million deaths in 2019, tuberculosis (TB) was registered as the deadliest infectious disease caused by a single pathogen surpassing HIV/AIDS. ${ }^{8}$ Meanwhile, the United Nations has made the elimination of TB one of its Sustainable Development Goals ${ }^{9}$ and the WHO End TB Strategy aims for a $90 \%$ reduction in TB incidence rate by $2035 .^{10}$ These global ambitions acknowledge the importance of community involvement in the fight to end TB, particularly through strengthening the community health workforce.

The contribution of CHWs to TB care and prevention have been well documented. ${ }^{11,12}$ Specifically, CHWs have enabled the task-shifting of a variety of TB program responsibilities to optimize capacity utilization of the public health system. These decentralized tasks include active case finding; ${ }^{13}$ sputum collection and transport, slide fixing and other laboratory support; ${ }^{14}$ and treatment support, such as communitybased directly observed treatment (DOT) and psychosocial support. ${ }^{15}$ As a result, the deployment of CHWs in TB programs has been linked to improved patient treatment outcomes, ${ }^{16}$ increased case notification, ${ }^{17}$ and decreased treatment loss to follow-up (LTFU). ${ }^{18}$ Nevertheless, despite the substantial evidence on the activities and responsibilities that $\mathrm{CHWs}$ can assume, the understanding of best practices for engagement of $\mathrm{CHWs}$ and the operationalization of $\mathrm{CHW}$ models for TB care and prevention remains limited.

Stop TB Partnership established TB REACH in 2010 to foster innovative approaches and technologies in TB care and prevention and catalyze their scale-up. Over the course of eight waves, the TB REACH mechanism ${ }^{19}$ has supported hundreds of projects utilizing numerous approaches to active case finding (ACF), many of which heeded the global call for greater involvement of $\mathrm{CHWs}$. As such, TB REACH represents one of the world's largest sources of funding and repository of information for interventions employing $\mathrm{CHWs}$ using a standardized monitoring and evaluation (M\&E) framework. ${ }^{20}$

\section{Objectives}

The objective of this study was to evaluate TB REACH projects on how they identified, engaged, paid, trained, supervised and evaluated CHWs for the purposes of ACF and the impact of employing specific 
practices for $\mathrm{CHW}$ engagement on project performance.

\section{Methods}

Study design

This was a cross-sectional study analyzing secondary data from the TB REACH project repository and primary data from subsequent project implementers surveys. We followed the Strengthening the Reporting of Observational studies in Epidemiology (STROBE) checklist for cross-sectional studies (www.strobe-statement.org), which is available in Additional File 1, Table A7: STROBE Checklist.

Project eligibility

We accessed the TB REACH Grant Management System (GMS) and included all projects from the first six TB REACH funding waves $(n=222)$ spanning from 2010 to 2020 . We excluded projects unrelated to ACF, did not utilize CHWs, had invalid contact information, or did not include the primary TB REACH impact indicator of "additionality" in TB case notification. ${ }^{20}$ In summary, this impact indicator is designed to capture the change in crude TB case notifications adjusted for trends in the pre-intervention period as well as in concurrent control areas.

Data sources

TB REACH project data repository

We reviewed project summaries, quarterly narrative reports and annual grant reviews from the GMS for eligible projects. A data collection template with 24 key project characteristics was created on the ONA platform (ONA Systems, Nairobi, Kenya) for systematic information extraction and abstraction. We extracted project characteristics and standard indicators for the TB care cascade. The data were verified by research staff, adjusted for consistency, and compiled into a single dataset.

Project implementer survey

We developed a 54-question survey based on current published literature and WHO guidelines ${ }^{4}$ to collect primary data on the CHW model employed by TB REACH grantees (see Additional File 1, Full Survey for Implementers). The survey instrument was provided through a web-based Google Forms survey and a Microsoft Word document. Survey flow and skip logic were built into the online survey and were clearly marked in the offline document. The full survey was sent to all primary contact persons of eligible projects. Secondary contacts were invited to complete the survey if the primary contact was unable to be reached or declined to participate. We requested participants to answer questions to the best of their ability and included an "unknown" response option to reduce recall bias. Anonymized data from the completed surveys were exported into Microsoft Excel. All survey responses were verified for completeness and consistency. The dataset was stored on a secure server and password protected with restricted access. To access the data, see Additional File 2. 
We calculated descriptive statistics for primary and secondary data. Additionality values from all projects included in the survey were summarized in a forest plot. We used random-effects meta-analysis model to calculate the pooled additionality. We used the additionality indicator to trifurcate eligible projects into groups of low, medium, and high impact. The bottom, low-impact (LI) group achieved an additionality of $\leq 5 \%$. The middle, medium-impact (MI) group consisted of projects that achieved an additionality of $>5 \%$ and $\leq 20 \%$. The top, high-impact $(\mathrm{HI})$ group reported an additionality of $>20 \%$. Differences in responses for these three impact groups were assessed using the ANOVA or Kruskal-Wallis test for continuous variables and the chi-squared or Fisher's exact test for categorical variables. We fitted linear randomeffect regressions to analyze the association between $\mathrm{CHW}$ factors and project additionality. We set the country of project implementation as the random effect. The primary outcome was the additionality indicator. The parameter coefficient $\left(\beta^{\prime}\right)$ was used to estimate the magnitude and direction of association. Univariate linear regressions were fitted between all possible $\mathrm{CHW}$ factors and additionality to identify significant factors to be included in the multivariate model (see Additional File 1, Table A1: Univariate association between community health worker activities and notification impact and Table A2: Univariate association between community health worker factors and project additionality per community health worker (percent additionality trend adjusted)). Covariates with a p-value $<0.05$ were included in the final model. Hypothesis tests were two-sided and point estimates included $95 \%$ confidence intervals $(\mathrm{Cl})$ for means and proportions, and inter-quartile ranges (IQR) for medians. Missing data or "unknown" responses were excluded from the analyses. Statistical analyses were performed on Stata v15 (StataCorp; College Station, TX, USA).

\section{Results}

Sample characteristics

The TB REACH repository included 222 projects (Fig. 1). After excluding ineligible projects due to lack of CHW engagement $(n=43)$, no ACF activities $(n=12)$, and/or missing project impact indicator $(n=7)$, we identified 123 eligible projects for invitation to the implementer's survey. We received a total of 57 survey responses over six weeks. Seven projects were excluded due to significant externalities confounding project additionality (see Additional File 1, Table A3: Factors Affecting Additionality) for a final sample of 50 projects from 24 countries (Fig. 2).

Pooled analysis provides a percentage additionality of $24 \cdot 0 \%(95 \% \mathrm{Cl} 21 \cdot 3 \%-26 \cdot 8 \%)$. The percentage additionality yield by projects ranged from $-16 \cdot 1 \%$ to $210 \cdot 8 \%$ showing substantial heterogeneity $\left(\mathrm{I}^{2}=\right.$ $99.9 \%$ ) (Fig. 3). When segmenting the sample by additionality into tertiles, we found an average additionality of $-2 \cdot 7 \%$ for $\mathrm{LI}$ projects, $11 \cdot 0 \%$ for $\mathrm{MI}$ projects, and $70 \cdot 7 \%$ for $\mathrm{HI}$ projects. The average grant duration was $19 \cdot 4$ months $(95 \% \mathrm{Cl} 18 \cdot 0-20 \cdot 9)$ with an average value of $\$ 502,345$ (95\% Cl $\$ 433,136$ $\$ 571,553)$. The 50 projects employed a total of $13,991 \mathrm{CHWs}$ with an average of $291 \mathrm{CHWs}$ per project (range: 4-3558). These CHWs detected 58,717 TB cases, an average of 1174 per project. Of those 
detected, 19,481 would not have been identified without ACF activities through these projects.

Approximately $68 \%$ of projects engaged $\mathrm{CHWs}$ to implement community-based active case finding, $6 \%$ employed CHWs in a health facility only, and $26 \%$ of projects had CHWs conduct activities in both settings. The high impact group had the highest mean number of total CHWs employed (311). $66 \%$ of projects were implemented in the African and Eastern Mediterranean WHO region, and 68\% (34/50) were conducted in lower-middle income countries (68\%).

Table 1: Characteristics of TB REACH projects associated with notification impact 
Table 1

Characteristics of TB REACH projects associated with notification impact

\begin{tabular}{|c|c|c|c|c|c|}
\hline & $\begin{array}{l}\text { LI } \\
(n=15)\end{array}$ & $\begin{array}{l}\text { MI } \\
(n=16)\end{array}$ & $\begin{array}{l}\text { HI } \\
(n=19)\end{array}$ & $\begin{array}{l}\text { Total } \\
(n=50)\end{array}$ & $\begin{array}{l}\text { p- } \\
\text { value }^{\square}\end{array}$ \\
\hline $\begin{array}{l}\% \text { trend-adjusted } \\
\text { additionality }\end{array}$ & $\begin{array}{l}0 \cdot 3(-7 \cdot 7- \\
3 \cdot 6)\end{array}$ & $\begin{array}{l}10(6 \cdot 8- \\
14 \cdot 4)\end{array}$ & $\begin{array}{l}56 \cdot 6(23 \cdot 9- \\
83 \cdot 2)\end{array}$ & $\begin{array}{l}12 \cdot 9(3 \cdot 8- \\
33 \cdot 6)\end{array}$ & <.001* \\
\hline $\begin{array}{l}\text { Average grant duration } \\
\text { (months) }\end{array}$ & $20 \cdot 8$ & $19 \cdot 9$ & $18 \cdot 0$ & $19 \cdot 4$ & $0 \cdot 268$ \\
\hline $\begin{array}{l}\text { Average grant value } \\
\text { (USD) }\end{array}$ & 546,133 & 509,739 & 461,548 & 502,345 & 0.606 \\
\hline \multicolumn{6}{|l|}{ Type of project } \\
\hline $\begin{array}{l}\text { Community-based active } \\
\text { case finding (ACF) only }\end{array}$ & $13(71 \%)$ & $9(69 \%)$ & $12(65 \%)$ & $34(68 \%)$ & \multirow[t]{3}{*}{$0 \cdot 353$} \\
\hline Facility-based ACF only & $0(0 \%)$ & $2(13 \%)$ & $1(6 \%)$ & $3(6 \%)$ & \\
\hline $\begin{array}{l}\text { Community \& Facility- } \\
\text { based ACF }\end{array}$ & $2(29 \%)$ & $5(19 \%)$ & $6(29 \%)$ & $13(26 \%)$ & \\
\hline 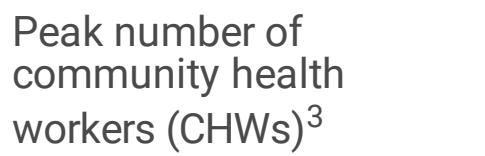 & $70(20-300)$ & $55(15-88)$ & $\begin{array}{l}86(17 \cdot 5- \\
300)\end{array}$ & $\begin{array}{l}60(17 \cdot 5- \\
276)\end{array}$ & $0.354^{*}$ \\
\hline Total number of $\mathrm{CHWs}^{2}$ & $\begin{array}{l}100(20- \\
450)\end{array}$ & $\begin{array}{l}60(25- \\
120)\end{array}$ & $86(17-86)$ & $76(20-425)$ & $0 \cdot 455^{\star}$ \\
\hline \multicolumn{6}{|l|}{ Country regions ${ }^{20}$} \\
\hline Africa & $7(48 \%)$ & $6(38 \%)$ & $8(42 \%)$ & $21(42 \%)$ & \multirow[t]{5}{*}{0.643} \\
\hline Americas & $2(13 \%)$ & $1(6 \%)$ & $0(0 \%)$ & $3(6 \%)$ & \\
\hline South-East Asia & $2(13 \%)$ & $4(25 \%)$ & $2(10 \%)$ & $8(16 \%)$ & \\
\hline Europe & $0(0 \%)$ & $1(6 \%)$ & $0(0 \%)$ & $1(2 \%)$ & \\
\hline Eastern Mediterranean & $2(13 \%)$ & $3(19 \%)$ & $7(37 \%)$ & $12(24 \%)$ & \\
\hline
\end{tabular}

$1: 1(2 \%)$ respondent missing information on questions asked

2: $2(4 \%)$ respondent missing information on questions asked

3: $5(10 \%)$ respondent missing information on questions asked

प: Fisher's exact test and chi-square tests: comparing proportions that is conditional on frequencies; ANOVA test: comparing means

*: Median and Kruskal-Wallis test 


\begin{tabular}{|c|c|c|c|c|c|}
\hline & $\begin{array}{l}\text { LI } \\
(n=15)\end{array}$ & $\begin{array}{l}\text { MI } \\
(n=16)\end{array}$ & $\begin{array}{l}\text { HI } \\
(n=19)\end{array}$ & $\begin{array}{l}\text { Total } \\
(n=50)\end{array}$ & $\begin{array}{l}\text { p- } \\
\text { value }^{\square}\end{array}$ \\
\hline Western Pacific & $2(13 \%)$ & $1(6 \%)$ & $2(10 \%)$ & $5(10 \%)$ & \\
\hline Project population & $3,769,353$ & $3,891,689$ & $1,443,028$ & $2,924,497$ & $0 \cdot 149$ \\
\hline \multicolumn{6}{|c|}{ Tuberculosis care cascade } \\
\hline $\begin{array}{l}\text { Number of people } \\
\text { verbally screened }\end{array}$ & $\begin{array}{l}150,918 \\
(24,629- \\
430,025)\end{array}$ & $\begin{array}{l}95,036 \\
(10,116- \\
335,536)\end{array}$ & $\begin{array}{l}175,837 \\
(91,093- \\
451,628)\end{array}$ & $\begin{array}{l}106,831 \\
(33,322- \\
365,601)\end{array}$ & $0 \cdot 258 *$ \\
\hline $\begin{array}{l}\text { Number of sputum } \\
\text { samples collected }\end{array}$ & $\begin{array}{l}7,714(3,784 \\
-19,562)\end{array}$ & $\begin{array}{l}6,972 \\
(1,592- \\
12,023)\end{array}$ & $\begin{array}{l}5,749 \\
(3,721- \\
9,705)\end{array}$ & $\begin{array}{l}6,391(3,531 \\
-12,082)\end{array}$ & $0 \cdot 443^{*}$ \\
\hline \multicolumn{6}{|c|}{ World Bank income classifications ${ }^{21}$} \\
\hline Low & $3(20 \%)$ & $6(38 \%)$ & $1(5 \%)$ & $10(20 \%)$ & \multirow[t]{3}{*}{$0 \cdot 131$} \\
\hline Lower-middle & $9(60 \%)$ & $9(56 \%)$ & $16(84 \%)$ & $34(68 \%)$ & \\
\hline Upper-middle & $3(20 \%)$ & $1(6 \%)$ & $2(11 \%)$ & $6(12 \%)$ & \\
\hline \multicolumn{6}{|c|}{$1: 1(2 \%)$ respondent missing information on questions asked } \\
\hline \multicolumn{6}{|c|}{ 2: $2(4 \%)$ respondent missing information on questions asked } \\
\hline \multicolumn{6}{|c|}{ 3: 5 (10\%) respondent missing information on questions asked } \\
\hline \multicolumn{6}{|c|}{$\begin{array}{l}\text { 口: Fisher's exact test and chi-square tests: comparing proportions that is conditional on frequencies; } \\
\text { ANOVA test: comparing means }\end{array}$} \\
\hline \multicolumn{6}{|c|}{ *: Median and Kruskal-Wallis test } \\
\hline
\end{tabular}

(See pages 28-29)

CHW model characteristics

Table 2 presents key characteristics of CHW models. Most projects (62\%) reported that CHWs only performed TB-related activities. For projects on which $\mathrm{CHWs}$ performed other tasks, CHWs spent an average of $80 \%$ of their time on TB-related activities. The majority of projects $(86 \%)$ had CHWs complete community outreach and verbal screening. Forty-three projects (88\%) provided CHWs with formal written contracts, and $33(77 \%)$ of those projects provided all their $\mathrm{CHWs}$ with undifferentiated contracts. Almost all projects conducted training using experts $(94 \%)$, while roughly half involved peers (54\%). Classroombased training was more commonly used than community-based training ( $96 \%$ vs $64 \%$ ). Electronic distance learning was used only by a minority of projects exclusively in the HI group (11\%). Thirty-one projects hosted periodic refresher trainings (65\%). 
Table 2

Key characteristics of the community health worker models associated with notification impact

$\begin{array}{lllll}\text { LI } & \text { MI } & \text { HI } & \text { Total } & \begin{array}{l}\text { p- } \\ (n=15)\end{array} \\ (n=16) & (n=19) & & \text { value }^{\square}\end{array}$

\section{Implementation activities}

Tuberculosis (TB) and other

$6(40 \%) \quad 5(31 \%) \quad 8(42 \%) \quad 19$

$(38 \%)$

$0 \cdot 790$

TB only

$9(60 \%) \quad 11(69 \%)$

11

31

(58\%) (62\%)

$\%$ time spent on TB activities

$77 \cdot 3$

85

$77 \cdot 9$

80

$0 \cdot 649$ *

Community outreach ${ }^{1}$

$13 / 15$

$13 / 15$

$16 / 19$

(84\%)

$42 / 49$

(86\%)

Verbal screening $^{1}$

(87\%)

(87\%)

$17 / 19$

(89\%)

$42 / 49$

(86\%)

HIV testing ${ }^{1}$

(87\%)

(80\%)

$2 / 19$

$1 / 15$
$(7 \%)$

$1 / 15$

(7\%)

(11\%)

$4 / 49$

$(8 \%)$

Sputum collection and transportation

12

(80\%)

11
$(73 \%)$

Linkage to treatment

Treatment counseling

$5(33 \%)$
$11(69 \%)$

$11(69 \%)$

13

(68\%)

$9(56 \%)$

\begin{tabular}{l}
$9(56 \%) \quad(79 \%)$ \\
\hline
\end{tabular}

13

$(68 \%)$
36

(72\%)

35

(70\%)

25

(50\%)

$1 \cdot 00$

$0 \cdot 730$

$1 \cdot 00$

$0 \cdot 712$

$0 \cdot 326$

$0 \cdot 106$

\section{Recruitment and selection}

Had prior experience ${ }^{2}$

$11 / 15$

(73\%)

$10 / 14$

(71\%)

$13 / 19$

$(68 \%)$

$34 / 48$

$(71 \%)$

0.951

Years of education ${ }^{3}$

12 (10-

14)

12 (10-

12)

10 (9-

12)

$12(10-$

12)

Provided written contracts

13

(87\%)

15 (94\%)

15

(79\%)

43

$(86 \%)$

From TB REACH

$7(54 \%)$

$13(87 \%)$

$8(53 \%)$

27

(65\%)

From non-governmental organization

$4(31 \%)$

$2(13 \%)$

$4(27 \%)$

10

(23\%)

From government

$2(15 \%)$

$1(7 \%)$

$3(20 \%)$

$6(14 \%)$

0.655

Provided differentiated contracts ${ }^{5}$

$10 / 12$

$(83 \%)$

$14 / 15$

(93\%)

$9 / 15$

$(60 \%)$

$33 / 42$

$(79 \%)$ 


\begin{tabular}{|c|c|c|c|c|c|}
\hline & $\begin{array}{l}\text { LI } \\
(n=15)\end{array}$ & $\begin{array}{l}\text { MI } \\
(n=16)\end{array}$ & $\begin{array}{l}\text { HI } \\
(n=19)\end{array}$ & Total & $\begin{array}{l}\text { p- } \\
\text { value }^{\square}\end{array}$ \\
\hline \multicolumn{6}{|l|}{ Training method } \\
\hline Expert & $\begin{array}{l}14 \\
(93 \%)\end{array}$ & $15(94 \%)$ & $\begin{array}{l}18 \\
(95 \%)\end{array}$ & $\begin{array}{l}47 \\
(94 \%)\end{array}$ & $1 \cdot 000$ \\
\hline Peer-to-peer & $\begin{array}{l}10 \\
(67 \%)\end{array}$ & $8(50 \%)$ & $9(47 \%)$ & $\begin{array}{l}27 \\
(54 \%)\end{array}$ & $0 \cdot 495$ \\
\hline Hands-on & $\begin{array}{l}14 \\
(93 \%)\end{array}$ & $13(81 \%)$ & $\begin{array}{l}17 \\
(90 \%)\end{array}$ & $\begin{array}{l}44 \\
(88 \%)\end{array}$ & 0.652 \\
\hline E-learning & $0(0 \%)$ & $0(0 \%)$ & $2(11 \%)$ & $2(4 \%)$ & $0 \cdot 323$ \\
\hline \multicolumn{6}{|l|}{ Training setting } \\
\hline Classroom-based & $\begin{array}{l}14 \\
(93 \%)\end{array}$ & $15(94 \%)$ & $\begin{array}{l}19 \\
(100 \%)\end{array}$ & $\begin{array}{l}48 \\
(96 \%)\end{array}$ & $0 \cdot 519$ \\
\hline Community-based & $8(53 \%)$ & $9(56 \%)$ & $\begin{array}{l}15 \\
(79 \%)\end{array}$ & $\begin{array}{l}32 \\
(64 \%)\end{array}$ & $0 \cdot 223$ \\
\hline Average hours of pre-service trainings ${ }^{4}$ & $\begin{array}{l}12(5- \\
24)\end{array}$ & $\begin{array}{l}12(8- \\
18)\end{array}$ & $\begin{array}{l}16(8- \\
30)\end{array}$ & $\begin{array}{l}16(8- \\
24)\end{array}$ & $0 \cdot 366^{*}$ \\
\hline \multicolumn{6}{|l|}{ Refresher training } \\
\hline Formal refresher trainings ${ }^{2}$ & $\begin{array}{l}9 / 13 \\
(69 \%)\end{array}$ & $\begin{array}{l}11 / 16 \\
(73 \%)\end{array}$ & $\begin{array}{l}11 / 19 \\
(58 \%)\end{array}$ & $\begin{array}{l}31 / 48 \\
(65 \%)\end{array}$ & $0 \cdot 646$ \\
\hline \multicolumn{6}{|l|}{ Formal training method $(n=31)$} \\
\hline Expert & $8(89 \%)$ & $\begin{array}{l}11 \\
(100 \%)\end{array}$ & $\begin{array}{l}10 \\
(91 \%)\end{array}$ & $\begin{array}{l}29 \\
(94 \%)\end{array}$ & $0 \cdot 740$ \\
\hline Peer-to-peer & $7(78 \%)$ & $10(91 \%)$ & $4(36 \%)$ & $\begin{array}{l}21 \\
(36 \%)\end{array}$ & 0.019 \\
\hline Hands-on & $6(67 \%)$ & $10(91 \%)$ & $8(73 \%)$ & $\begin{array}{l}24 \\
(77 \%)\end{array}$ & $0 \cdot 437$ \\
\hline E-learning & $0(0 \%)$ & $0(0 \%)$ & $2(18 \%)$ & $2(7 \%)$ & $0 \cdot 314$ \\
\hline \multicolumn{6}{|l|}{ Formal training setting } \\
\hline Classroom-based & $8(89 \%)$ & $10(91 \%)$ & $7(64 \%)$ & $\begin{array}{l}25 \\
(81 \%)\end{array}$ & $0 \cdot 205$ \\
\hline Community-based & $4(44 \%)$ & $6(55 \%)$ & $7(64 \%)$ & $\begin{array}{l}17 \\
(55 \%)\end{array}$ & $0 \cdot 692$ \\
\hline Frequency of refresher trainings ${ }^{4}$ & $3(1-4)$ & $2(2-4)$ & $2(2-4)$ & $2(2-4)$ & $0.978 *$ \\
\hline
\end{tabular}




\begin{tabular}{|c|c|c|c|c|c|}
\hline & $\begin{array}{l}\text { LI } \\
(n=15)\end{array}$ & $\begin{array}{l}\text { MI } \\
(n=16)\end{array}$ & $\begin{array}{l}\text { HI } \\
(n=19)\end{array}$ & Total & $\begin{array}{l}\text { p- } \\
\text { value }^{\square}\end{array}$ \\
\hline Average hours of refresher trainings ${ }^{3}$ & $4(3-8)$ & $5(3-8)$ & $7(3-8)$ & $\begin{array}{l}5 \cdot 5(3- \\
8)\end{array}$ & $0 \cdot 725^{\star}$ \\
\hline \multicolumn{6}{|l|}{ Supervision } \\
\hline Issues addressed by direct supervisor ${ }^{4}$ & $\begin{array}{l}13 / 15 \\
(87 \%)\end{array}$ & $\begin{array}{l}13 / 13 \\
(100 \%)\end{array}$ & $\begin{array}{l}16 / 19 \\
(84 \%)\end{array}$ & $\begin{array}{l}42 / 47 \\
(89 \%)\end{array}$ & $0 \cdot 420$ \\
\hline Issues addressed by upper management ${ }^{4}$ & $\begin{array}{l}3 / 15 \\
(20 \%)\end{array}$ & $\begin{array}{l}0 / 13 \\
(0 \%)\end{array}$ & $\begin{array}{l}4 / 19 \\
(22 \%)\end{array}$ & $\begin{array}{l}7 / 47 \\
(15 \%)\end{array}$ & $0 \cdot 197$ \\
\hline Female supervisor $(\%)^{2}$ & $58 \cdot 8$ & $53 \cdot 5$ & $29 \cdot 5$ & $45 \cdot 6$ & 0.007 \\
\hline $\begin{array}{l}\text { Average \# community health workers } \\
(\mathrm{CHWs}) \text { per supervisor }{ }^{3}\end{array}$ & $\begin{array}{l}13(7- \\
26)\end{array}$ & $7(6-10)$ & $\begin{array}{l}15(5- \\
60)\end{array}$ & $\begin{array}{l}9(6- \\
25)\end{array}$ & $0 \cdot 247 *$ \\
\hline $\begin{array}{l}\text { Average \# of supervisor reviews per } \\
\text { quarter }^{2}\end{array}$ & $\begin{array}{l}9(3- \\
12)\end{array}$ & $6(3-12)$ & $\begin{array}{l}6(3- \\
12)\end{array}$ & $\begin{array}{l}6(3- \\
12)\end{array}$ & $0 \cdot 775^{\star}$ \\
\hline $\begin{array}{l}\text { Average \# of supervisor direct feedback } \\
\text { per quarter }^{2}\end{array}$ & $\begin{array}{l}9(3- \\
12)\end{array}$ & $3(1-12)$ & $\begin{array}{l}6(4- \\
12)\end{array}$ & $\begin{array}{l}6(3- \\
12)\end{array}$ & $0 \cdot 293^{*}$ \\
\hline \multicolumn{6}{|l|}{ Sustainability and integration } \\
\hline Promoted to a higher role & $\begin{array}{l}5 / 14 \\
(36 \%)\end{array}$ & $\begin{array}{l}8 / 13 \\
(62 \%)\end{array}$ & $\begin{array}{l}8 / 19 \\
(42 \%)\end{array}$ & $\begin{array}{l}21 / 46 \\
(46 \%)\end{array}$ & $0 \cdot 426$ \\
\hline \multicolumn{6}{|c|}{$\mathrm{CHWs}$ working on the project keep their jobs at the close of the project ${ }^{2}$} \\
\hline All kept their jobs after project & $\begin{array}{l}7 / 14 \\
(50 \%)\end{array}$ & $\begin{array}{l}1 / 15 \\
(7 \%)\end{array}$ & $\begin{array}{l}5 / 19 \\
(26 \%)\end{array}$ & $\begin{array}{l}13 / 48 \\
(27 \%)\end{array}$ & 0.064 \\
\hline A subset kept their jobs after project & $\begin{array}{l}4 / 14 \\
(29 \%)\end{array}$ & $\begin{array}{l}11 / 15 \\
(73 \%)\end{array}$ & $\begin{array}{l}11 / 19 \\
(58 \%)\end{array}$ & $\begin{array}{l}26 / 48 \\
(54 \%)\end{array}$ & \\
\hline None kept their jobs after project & $\begin{array}{l}4 / 14 \\
(29 \%)\end{array}$ & $\begin{array}{l}2 / 15 \\
(13 \%)\end{array}$ & $\begin{array}{l}3 / 19 \\
(16 \%)\end{array}$ & $\begin{array}{l}9 / 48 \\
(19 \%)\end{array}$ & \\
\hline $\begin{array}{l}\text { Continued with the same responsibilities } \\
(\mathrm{N}=39)\end{array}$ & $\begin{array}{l}8 / 11 \\
(73 \%)\end{array}$ & $\begin{array}{l}3 / 12 \\
(25 \%)\end{array}$ & $\begin{array}{l}13 / 16 \\
(81 \%)\end{array}$ & $\begin{array}{l}24 / 39 \\
(62 \%)\end{array}$ & 0.007 \\
\hline \multicolumn{6}{|c|}{$\begin{array}{l}\text { Data are \%, mean or median. \% are calculated based on the total number of projects with available } \\
\text { data. Percentages within each category are based on the total projects within each category. } \mathrm{N} \text { sizes } \\
\text { are listed for variables with missing values. }\end{array}$} \\
\hline \multicolumn{6}{|c|}{ 1: 1 (2\%) respondent missing information on questions asked } \\
\hline \multicolumn{6}{|c|}{ 2: 2 (4\%) respondents missing information on questions asked } \\
\hline
\end{tabular}



LI MI
HI
Total
p-
$(n=15) \quad(n=16) \quad(n=19)$
value $^{\square}$

4: $3(6 \%)$ respondents missing information on questions asked

5: 8 (16\%) respondents missing information on questions asked

प: Fisher's exact test and chi-square tests: comparing proportions that is conditional on frequencies; ANOVA test: comparing means

*: Median (IQR) and Kruskal-Wallis test

9 : As indicated by WHO CHW training guidelines, expert, peer-to-peer, and hands-on training indicates face-to-face interaction as opposed to distance learning (E-learning). Classroom-based training emphasizes theoretical knowledge; community-based training emphasizes practical application.

Most projects cited that CHW performance issues were addressed by a direct supervisor (89\%) rather than by upper management (15\%). Projects employed slightly more male supervisors on average (54.4\% male vs $45.6 \%$ female). Each supervisor oversaw an average of $33 \mathrm{CHWs}$, with a higher average in the high impact group (60). All or a subset of CHWs kept their jobs after the TB REACH project ended in $81 \%$ of projects.

There were select notable differences between the three impact groups. There was an inverse relationship between the number of projects that provided their $\mathrm{CHWs}$ with the same contracts rather than differentiated labor agreements. We measured a higher proportion of projects using community-based training in the $\mathrm{HI}$ group, while lower impact projects completed more peer-to-peer training (see Additional File 1, Table A6: Differentiated contracts stratified by impact level). Projects in the HI category employed male supervisors significantly more frequently. Finally, there was a significant relationship between $\mathrm{CHWs}$ continuing with the same responsibilities after the close of a project and project impact $(p=0.007)$.

\section{Table 2: Key characteristics of the community health worker models associated with notification impact}

(See pages 29-31)

Table 3 shows the results of the univariate and multivariate linear regression. Full results from the univariate analysis are listed in Additional File 1, Table A3: Factors Affecting Additionality. Compared with projects that did not provide pre-service training through e-learning, projects that provided e-learning ( $\beta$ $=98.44, p<0.001)$ were associated with higher additionality. An increase of one CHW per supervisor is associated with a 0.35 increase in the project's additionality. 
Table 3

Associations between community health worker factors and project's trend adjusted percent additionality

\begin{tabular}{|c|c|c|c|c|c|c|}
\hline & \multicolumn{3}{|c|}{ Univariate analysis $(\mathrm{N}=50)$} & \multicolumn{3}{|c|}{ Multivariate analysis $(\mathrm{N}=41)$} \\
\hline & $\mathbf{n}$ & Coef. (95\% Cl) & $\begin{array}{l}\mathrm{p}- \\
\text { value }\end{array}$ & $\mathbf{n}$ & Coef. (95\% Cl) & $\begin{array}{l}\mathrm{p}- \\
\text { value }\end{array}$ \\
\hline Peak number of $\mathrm{CHWs}$ & 45 & $0.05(0.01-0.09)$ & 0.022 & 41 & $\begin{array}{l}0.005(-0.04- \\
0.05)\end{array}$ & $0 \cdot 817$ \\
\hline Total number of $\mathrm{CHWs}$ & 48 & $0.03(0.01-0.05)$ & 0.013 & 41 & $\begin{array}{l}0.008(-0.01- \\
0.03)\end{array}$ & $0 \cdot 482$ \\
\hline \multicolumn{7}{|c|}{ Pre-service training - E-learning } \\
\hline Yes & 2 & $\begin{array}{l}94 \cdot 83(29 \cdot 65- \\
160 \cdot 01)\end{array}$ & 0.005 & 2 & $\begin{array}{l}98 \cdot 44(44 \cdot 82- \\
152 \cdot 06)\end{array}$ & $<.001$ \\
\hline No & 48 & ref & & 39 & & \\
\hline $\begin{array}{l}\text { Average CHWs per } \\
\text { supervisor }\end{array}$ & 44 & $0.38(0.20-0.57)$ & $<.001$ & 41 & $0.35(0.12-0.58)$ & 0.002 \\
\hline CHW compensation & & & & & & \\
\hline
\end{tabular}

Table 4 details compensation schemes employed on the TB REACH projects. Thirty-seven projects (74\%) included or utilized a fixed component in their compensation, while $56 \%$ included or utilized variable compensation. The average total remuneration was US\$153.31 per month. The average fixed component per month was US\$124.44 and the average variable component per month was US\$28.87. A breakdown of CHW compensation by income group and World Bank country income classification is provided in Additional File 1, Table A4: CHW compensation by fixed and variable component and World Bank country classification and Additional File 1, Table A5: CHWs compensation by World Bank country classification.

There was no significant difference in total, fixed or variable compensation across the three impact groups. The greatest average total compensation per month was provided by the MI group (US\$201.62); the total compensation by $\mathrm{LI}$ and $\mathrm{HI}$ groups were comparable (US\$127.77 and US\$153.32, respectively). There was a larger proportion of projects from the $\mathrm{HI}$ and $\mathrm{Ml}$ groups where $\mathrm{CHWs}$ were given priority access to disease testing $(p=0 \cdot 294)$. 
Table 4

Monetary and non-monetary compensation of community health workers associated with notification impact

$\begin{array}{lllll}\text { LI } & \text { MI } & \text { HI } & \text { Total } & \text { p- } \\ (n=15) & (n=16) & (n=19) & (n=50) & \text { value }^{\square}\end{array}$

Monetary compensation

Earned only a fixed component (salary or $\quad 5(33 \%) \quad 6(38 \%) \quad 7(37 \%) \quad 18(36 \%) \quad 0.783$ stipend)

Earned only a variable component $\quad 3(20 \%) \quad 3(19 \%) \quad 2(11 \%) \quad 8(16 \%)$

(incentives) $^{1}$

Earned both a fixed and variable $\quad 5(33 \%) \quad 7(44 \%) \quad 7(37 \%) \quad 19(38 \%)$

component

No monetary compensation

$2(13 \%) \quad 0(0 \%) \quad 3(16 \%) \quad 5(10 \%)$

Average total compensation/month

$127 \cdot 77$

$210 \cdot 62$

$127 \cdot 51$

$153 \cdot 31$

$0 \cdot 106$

$(\text { USD })^{2}$

Average fixed compensation/month (USD)

$97 \cdot 31$

$172 \cdot 31$

$107 \cdot 61$

$124 \cdot 44$

$0 \cdot 219$

Average variable compensation/month

$30 \cdot 46$

$38 \cdot 31$

$19 \cdot 91$

$28 \cdot 87$

0.523

(USD)

\section{Non-monetary incentives ${ }^{3}$}

Priority access to TB/HIV or other disease testing

$3 / 13$

(23\%)

Health insurance
$2 / 13$

(15\%)
$4 / 12$

(33\%)

$1 / 11$

(9\%)
$9 / 18$

(50\%)

$4 / 19$

(22\%)
$16 / 43$

(37\%)

$7 / 43$

(17\%)

$\mathrm{N}$ sizes are listed for variables with missing values.

${ }^{1}: 2(4 \%)$ respondent missing information on questions asked

2: $8(16 \%)$ respondent missing information on questions asked

3. 7 (14\%) respondent missing information on questions asked

${ }^{\square}$ Fisher's exact test and chi-square tests: comparing proportions that is conditional on frequencies; ANOVA test: comparing means

\section{Discussion}

Our retrospective data collection and analysis of 50 survey responses from TB REACH project implementers provided information on a broad range of $\mathrm{CHW}$ engagement models for TB interventions 
carried out globally. This information contributes to filling the knowledge gaps in the existing literature on the roles of $\mathrm{CHWs}$ and elucidates variables facilitating project success. The results of our study found that $\mathrm{CHW}$ interventions overall had a large positive impact on yielding incremental TB notifications in intervention areas. The $\mathrm{CHW}$ model factors more likely to influence project outcomes are the training method and setting, supervisory models, and integration into the existing health system.

Regarding training, we found capacity building through hands-on or practical applications in a community setting was associated with more successful case finding. This was concordant with a review that found training for diagnostic tests should be addressed through hands-on practice and consistent feedback from experienced health care workers or supervisors. ${ }^{23}$ Training completed in the community is also highlighted by Gilroy and Winch, in which a commonly cited problem in $\mathrm{CHW}$ studies is "training that is too classroom-based with little practical hands-on experience". ${ }^{24}$ While our survey found that the classroom was the most common training setting, some projects reported training in both the classroom and the community. The higher impact groups had a larger proportion of projects that incorporated community-based training. This reinforces that training $\mathrm{CHWs}$ on practical skills in the community which they will eventually serve can contribute to better prepared health workers.

Our multivariate regression found that the two projects utilizing e-learning methods during pre-service training yielded higher additionality. Although e-learning is not yet commonly used for $\mathrm{CHW}$ training, the current COVID-19 pandemic may catalyze a potentially positive paradigm shift in capacity building. A study in Sub-Saharan Africa found that the blended approach combining e-learning with classroom training for $\mathrm{CHWs}$ improved standardization of training content and retention to course materials due to the ability to revisit online course materials. ${ }^{25}$ The study also showed a total training cost savings of $42 \%$ from reduced travel, personnel, and classroom costs. Thus, e-learning proves to be a promising mode of training. Further studies and projects should explore the potential of integrating mobile or remote health technologies in their training models, especially given the new realities of travel and meetings in many countries.

The majority of projects in our survey had $\mathrm{CHW}$ performance issues addressed by a direct supervisor without the need of upper management involvement. Supervisors provided direct feedback to $\mathrm{CHWs}$ an average of 13 times per quarter (three months), leading us to believe that the regularity of feedback is unlikely to influence project outcomes. However, a previous study on CHW models have also noted that regular supervision can be demotivating as some $\mathrm{CHWs}$ perceive their supervision to be linked to their poor performance. ${ }^{26}$ Conversely, more supportive supervision by formal health workers can provide CHWs with a sense of legitimacy and increased work motivation. ${ }^{27}$ As the evidence base remains ambiguous, more research is needed on the most optimal roles of supervisors should play in order to best support CHWs.

The effective integration of CHWs into the existing health systems is a well-documented enabler of project success 5,6 as it enhances sustainability and fosters collaboration between lay and formal healthcare providers. CHWs have also vociferously noted an enhanced credibility and clarity of their own 
roles with a greater level of integration. ${ }^{5,28}$ Our study results were concordant with these findings. Projects that had CHWs continue the same roles and responsibilities after the close of the project tended to produce a greater additionality impact on TB notifications. This may suggest that projects offering the prospect of long-term engagement through ties with the public health system and other health networks had a positive impact on $\mathrm{CHW}$ performance. Another hypothesis is that $\mathrm{CHWs}$ originally employed by the project decided to continue their roles because the project was successful. Pallas et al. posit that integrating $\mathrm{CHW}$ programs into the agendas of ministries of health, non-governmental organizations, and international donors can create more impactful $\mathrm{CHW}$ models that are sustainable even during times of political unrest or a reduction of external donor funding. ${ }^{29}$ Thus, it is beneficial to embed CHW models within primary health care systems for them to be both sustainable and impactful.

More projects that we surveyed compensated their $\mathrm{CHWs}$ through a fixed component (salary or stipend) than through a variable component. A study on three large CHW programs in India has found an unmet demand for salaried positions and service conditions for $\mathrm{CHWs}$ and that the volunteer status can hinder $\mathrm{CHWs}$ from receiving job benefits and fair salaries. This study specified that performance-based incentives lack financial security in large scale CHW programs such as the Accredited Social Health Activist (ASHA). ${ }^{30}$ Moreover, our survey also evinced that more projects in the high impact group tended to provide their $\mathrm{CHWs}$ with additional benefits beyond monetary compensation, including the provision of health insurance and priority access to disease testing. This highlights that additional benefits contribute to higher $\mathrm{CHW}$ job satisfaction and performance, which in turn may translate into to higher outputs and larger impact of the project. The importance of fair and commensurate compensation and the potentially positive impact on TB case notifications has been noted in other studies as well. ${ }^{31}$

A key strength of this study was the unique data source. As one of the largest funding mechanisms for TB, our access to the GMS allowed us to use a robust dataset on the characteristics and outcomes of projects with $\mathrm{CHW}$ engagement models across many different countries. Access to this database also facilitated the recruitment of project implementers. Our survey was developed to closely align with the WHO guidelines on health policy and system support to optimize CHW programs, allowing us to obtain details on how concrete implementers' experiences support or discount the recommendations of these guidelines, and which of the recommendations to prioritize in the context of leveraging CHWs for TB ACF. As such, while our study results are largely concordant with WHO recommendations, concrete experiences from TB REACH projects utilizing CHWs identified factors such as community-based training, rigorous support and supervision, and intentional integration into the existing health care system that may be prioritized to increase the likelihood of incremental notification impact and successful implementation of CHW models for TB.

Our study was limited by the attempt to associate project and CHW characteristics with TB REACH's primary outcome metric, trend-adjusted changes in TB case notifications (additionality). While this can offer a less biased measure of performance, we had to exclude a number of projects for which the additionality assessment was confounded by external factors (see Additional File 1, Table A3: Factors Affecting Additionality). Additionality is also only one metric by which active TB case finding 
interventions can be assessed, and it is possible that projects that yielded a low additionality could have had a high impact on other evaluation metrics. We also experienced a low overall response rate $(41 \%)$ to our survey. Several implementers noted that responses to specific survey questions may not be accurate due to difficulties recalling details from projects implemented many years ago.

\section{Conclusion}

Interventions that use $\mathrm{CHWs}$ documented large increases in the number of people treated for TB in project areas. This study elucidated possible factors that have a larger impact on TB ACF projects using CHWs. In line with the 2018 WHO guidelines for CHW models, this study highlights that $\mathrm{CHWs}$ should be provided with ongoing dynamic types of training, supportive and continuous supervision, and fair compensation as well as be well-integrated into the existing primary healthcare system. These areas may be prioritized when designing TB projects with a $\mathrm{CHW}$ engagement component in order to enhance the potential for a higher notification impact and more sustainable engagement of $\mathrm{CHWs}$.

\section{Abbreviations}

\begin{tabular}{ll} 
ACF & Active case finding \\
\hline CHW & Community Healthcare Worker \\
\hline DOT & Directly observed treatment \\
\hline GMS & Grant management system \\
\hline HI & High-impact \\
\hline HIV & Human immunodeficiency virus \\
\hline IQR & Inter-quartile ranges \\
\hline LI & Low-impact \\
\hline LTFU & Loss to follow-up \\
\hline M\&E & Monitoring \& Evaluation \\
\hline MI & Medium-impact \\
\hline STROBE & Strengthening the Reporting of Observational studies in Epidemiology \\
\hline TB & Tuberculosis \\
\hline WHO & World Health Organization \\
\hline
\end{tabular}

\section{Declarations}

\section{Ethics approval and consent to participate}


Ethical approval for this study was granted by the Ethics Committee for Biomedical Research of the Ha Noi School of Public Health (511/2019/YTCC-HD3). All participating respondents were given a participant information sheet and signed an informed consent form prior to completing the survey. We removed all personally-identifiable information from the survey responses prior to the analyses.

\section{Consent for publication}

Consent for publication was not obtained because de-identified data were used and presented in this manuscript.

\section{Availability of data and materials}

All data generated or analyzed during this study are included in this published article and its supplementary information files.

\section{Competing interests}

The authors declare that they have no competing interests.

\section{Funding}

TB REACH is supported by Global Affairs Canada through grant number CA-3-D000920001. https://w05.international.gc.ca/projectbrowser-banqueprojets/projectprojet/ details/d000920001. Funders were not involved in the design of the study or collection, analysis, and interpretation of data or in writing the manuscript.

\section{Author's contributions}

TD conducted the literature search, data curation, formal analysis, data validation and visualization. RF assisted with study conceptualization, performed data curation, investigation, input on methodology, project administration, supervision, validation, and data visualization. PT conducted data curation, data validation, formal analysis, insight into methodology and data visualization. LV conceptualized the study, reviewed the methodology, conducted project administration, supervision, data validation and visualization. AC helped conceptualize the study and assisted with developing the methodology, data visualization and figures. LN assisted with study conceptualization and design. JC was the driving force behind the study's conceptualization. He provided data curation, contextualization and data visualization. TD, RF and PT accessed and verified the underlying data. TD and PT drafted the first version of the 
manuscript. All authors reviewed and edited the manuscript. All authors read and approved the final manuscript.

\section{Acknowledgements}

We express our sincere gratitude to the project implementers who participated in the survey and to the projects and CHWs that they represented, which detected thousands of people with TB.

\section{References}

1. World Health Organization. Declaration of Alma Ata [Internet]. Geneva: World Health Organization; 2018 [cited 2020 Nov 28]. Available from: https://www.who.int/publications/almaata_declaration_en.pdf.

2. Lancet T. The Astana Declaration: the future of primary health care? The Lancet. 2018 Oct 20;392(10156):1369.

3. World Health Organization. Global strategy on human resources for health: workforce 2030 [Internet]. World Health Organization; 2016 [cited 2021 Aug 18]. 64 p. Available from: https://apps.who.int/iris/handle/10665/250368.

4. World Health Organization. WHO guideline on health policy and system support to optimize community health worker programmes. [Internet]. 2018 [cited 2020 Nov 28]. Available from: http://www.ncbi.nlm.nih.gov/books/NBK533329/.

5. Scott K, Beckham SW, Gross M, et al. What do we know about community-based health worker programs? A systematic review of existing reviews on community health workers. Hum Resour Health [Internet]. 2018 Aug 16 [cited 2020 Nov 28];16. Available from: https://www.ncbi.nlm.nih.gov/pmc/articles/PMC6097220/.

6. Kok MC, Dieleman M, Taegtmeyer M, et al. Which intervention design factors influence performance of community health workers in low- and middle-income countries? A systematic review. Health Policy Plan. 2015 Nov;30(9):1207-27.

7. Perry HB, Rassekh BM, Gupta S, Wilhelm J, Freeman PA. Comprehensive review of the evidence regarding the effectiveness of community-based primary health care in improving maternal, neonatal and child health: 1 . rationale, methods and database description. J Glob Health [Internet]. [cited 2020 Nov 28];7(1). Available from: https://www.ncbi.nlm.nih.gov/pmc/articles/PMC5491943/.

8. WHO |. Global tuberculosis report 2019 [Internet]. WHO. [cited 2020 Feb 4]. Available from: http://www.who.int/tb/publications/global_report/en/.

9. Health - United. Nations Sustainable Development [Internet]. [cited 2020 Nov 28]. Available from: https://www.un.org/sustainabledevelopment/health/.

10. WHO | WHO End TB Strategy [Internet]. WHO. [cited 2019 Apr 17]. Available from: https://www.who.int/tb/post2015_strategy/en/. 
11. Mhimbira FA, Cuevas LE, Dacombe R, Mkopi A, Sinclair D. Interventions to increase tuberculosis case detection at primary healthcare or community-level services. Cochrane Database Syst Rev [Internet]. 2017 [cited 2020 Dec 7];(11). Available from:

https://www.cochranelibrary.com/cdsr/doi/10.1002/14651858.CD011432.pub2/abstract? cookiesEnabled.

12. StopTB Field guide 3: Finding missing people with TB in communities [Internet]. the Stop TB Partnership, hosted by the United Nations Office for Project Services; 2018. Available from: https://stoptb-strategicinitiative.org/elearning/wp-content/uploads/2019/04/STBFG_03.pdf.

13. Maung HMW, Saw $S$, Isaakidis $P$, et al. The contribution of a non-governmental organisation's Community Based Tuberculosis Care Programme to case finding in Myanmar: trend over time. Infect Dis Poverty. 2017 Apr;3(1):51. 6(.

14. Datiko DG, Yassin MA, Theobald SJ, et al. Health extension workers improve tuberculosis case finding and treatment outcome in Ethiopia: a large-scale implementation study. BMJ Glob Health. 2017 Nov 1;2(4):e000390.

15. Li X, Wang B, Tan D, et al. Effectiveness of comprehensive social support interventions among elderly patients with tuberculosis in communities in China: a community-based trial. J Epidemiol Community Health. 2018 May;72(5):369-75.

16. Vo LNQ, Codlin AJ, Forse RJ, et al. Tuberculosis among economic migrants: a cross-sectional study of the risk of poor treatment outcomes and impact of a treatment adherence intervention among temporary residents in an urban district in Ho Chi Minh City, Viet Nam. BMC Infect Dis. 2020 Feb 12;20(1):134.

17. Mac TH, Phan TH, Nguyen VV, et al. Optimizing Active Tuberculosis Case Finding: Evaluating the Impact of Community Referral for Chest X-ray Screening and Xpert Testing on Case Notifications in Two Cities in Viet Nam. Trop Med Infect Dis. 2020 Dec;5(4):181.

18. Mitnick C, Bayona J, Palacios E, et al. Community-Based Therapy for Multidrug-Resistant Tuberculosis in Lima, Peru. N Engl J Med. 2003 Jan;9(2):119-28. 348(.

19. Creswell J, Khan A, Bakker Ml, et al. The TB REACH Initiative: Supporting TB Elimination Efforts in the Asia-Pacific. Trop Med Infect Dis. 2020 Dec;5(4):164.

20. Blok L, Creswell J, Stevens R, et al. A pragmatic approach to measuring, monitoring and evaluating interventions for improved tuberculosis case detection. Int Health. 2014 Sep;6(3):181-8.

21. World Health Organization. World health statistics 2011. Geneva: World Health Organization; 2011.

22. World Bank Country and Lending Groups. - World Bank Data Help Desk [Internet]. [cited 2020 Nov 29]. Available from: https://datahelpdesk.worldbank.org/knowledgebase/articles/906519.

23. Ruizendaal E, Dierickx S, Peeters Grietens K, Schallig HD, Pagnoni F, Mens PF. Success or failure of critical steps in community case management of malaria with rapid diagnostic tests: a systematic review. Malar J. 2014 Jun;12(1):229. 13(.

24. 24. 
25. Sissine M, Segan R, Taylor M, et al. Cost Comparison Model: Blended eLearning versus traditional training of community health workers. Online J Public Health Inform [Internet]. 2014 Dec 15 [cited 2021 Jan 18];6(3). Available from: https://www.ncbi.nlm.nih.gov/pmc/articles/PMC4292533/.

26. Stekelenburg J, Kyanamina SS, Wolffers I. Poor performance of community health workers in Kalabo District, Zambia. Health Policy. 2003 Aug 1;65(2):109-18.

27. Kane SS, Gerretsen B, Scherpbier R, Dal Poz M, Dieleman M. A realist synthesis of randomised control trials involving use of community health workers for delivering child health interventions in low and middle income countries. BMC Health Serv Res. 2010 Oct 13;10(1):286.

28. Biermann O, Dixit K, Rai B, Caws M, Lönnroth K, Viney K. Building on facilitators and overcoming barriers to implement active tuberculosis case-finding in Nepal, experiences of community health workers and people with tuberculosis. BMC Health Serv Res. 2021 Apr 1;21(1):295.

29. Pallas SW, Minhas D, Pérez-Escamilla R, Taylor L, Curry L, Bradley EH. Community health workers in low- and middle-income countries: what do we know about scaling up and sustainability? Am J Public Health. 2013 Jul;103(7):e74-82.

30. Bhatia K. Community health worker programs in India: a rights-based review. Perspect Public Health. 2014 Sep;134(5):276-82.

31. Vo LNQ, Forse RJ, Codlin AJ, et al. A comparative impact evaluation of two human resource models for community-based active tuberculosis case finding in Ho Chi Minh City, Viet Nam. BMC Public Health. 2020 Jun 15;20(1):934.

\section{Figures}




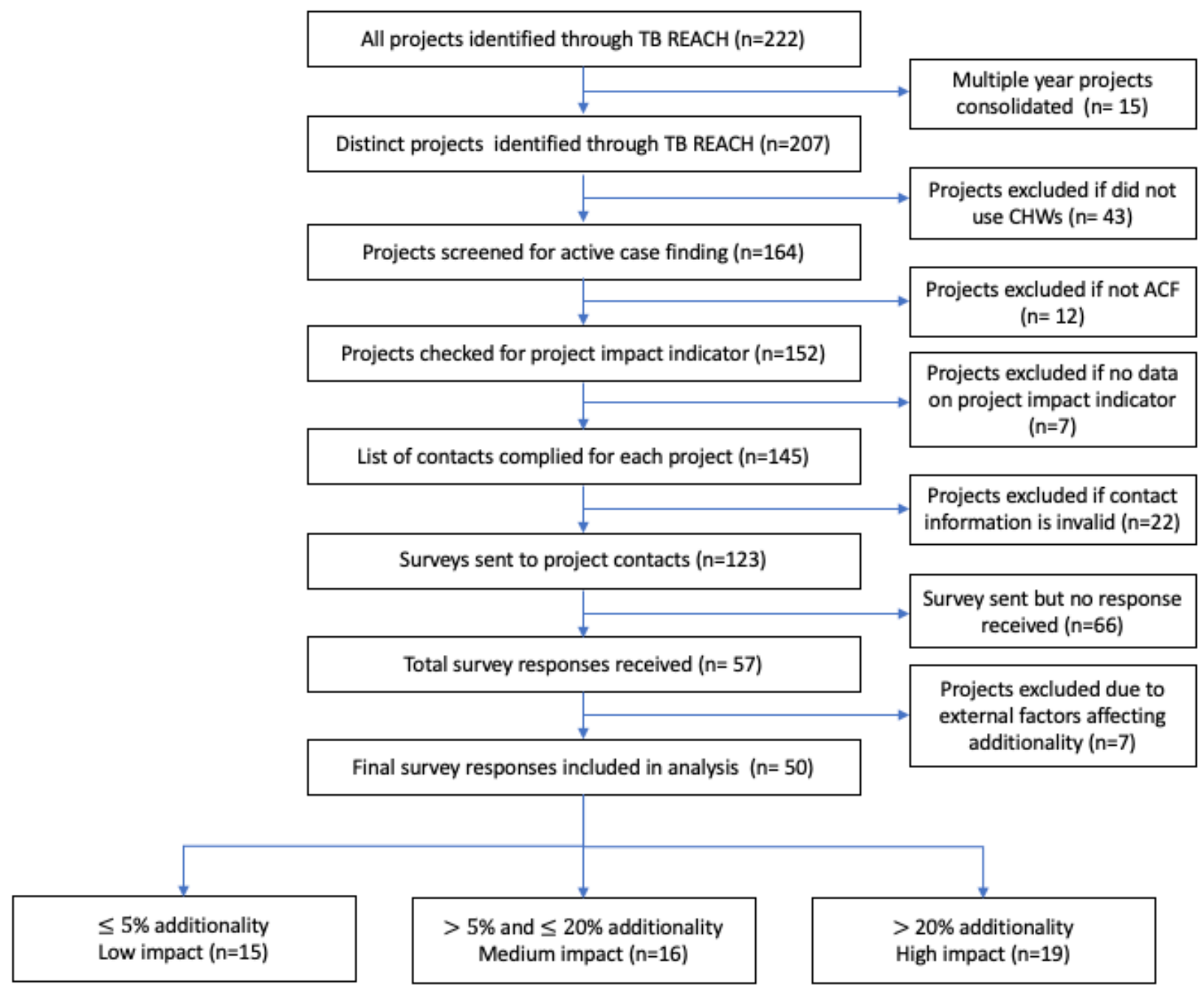

\section{Figure 1}

Project selection flow chart

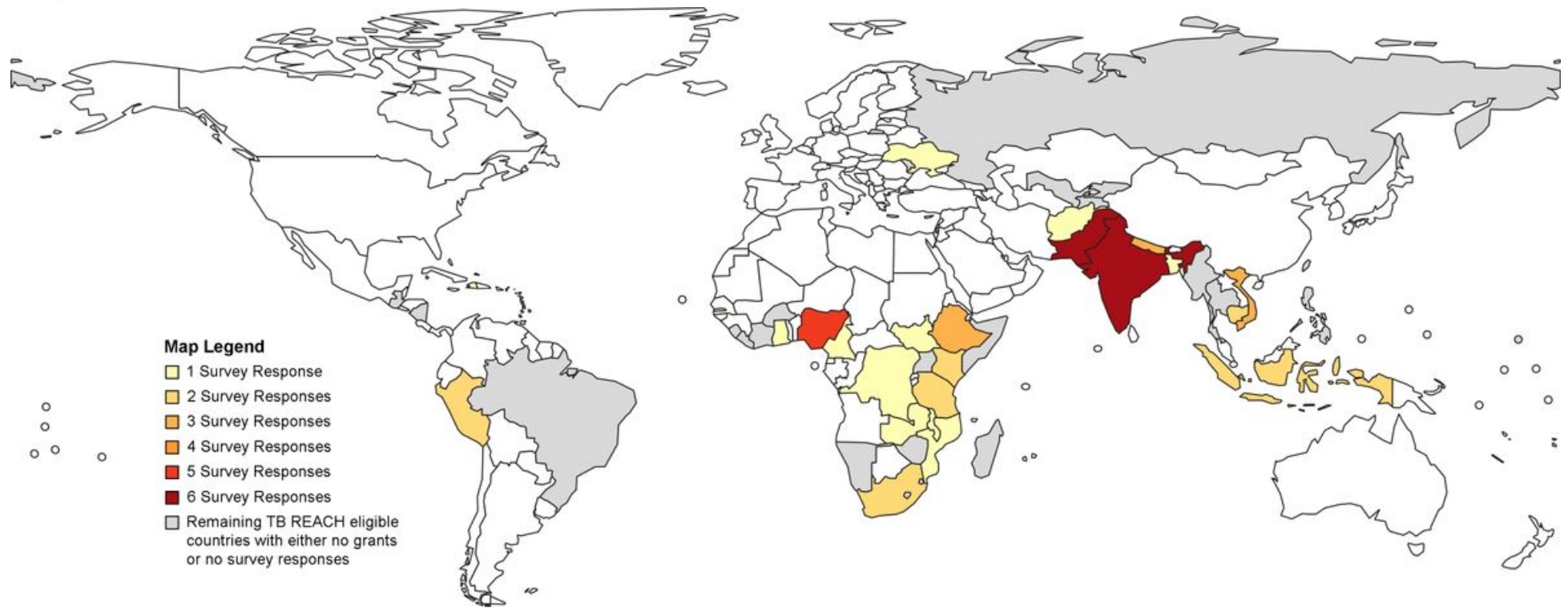

Figure 2 
World map of TB REACH project countries

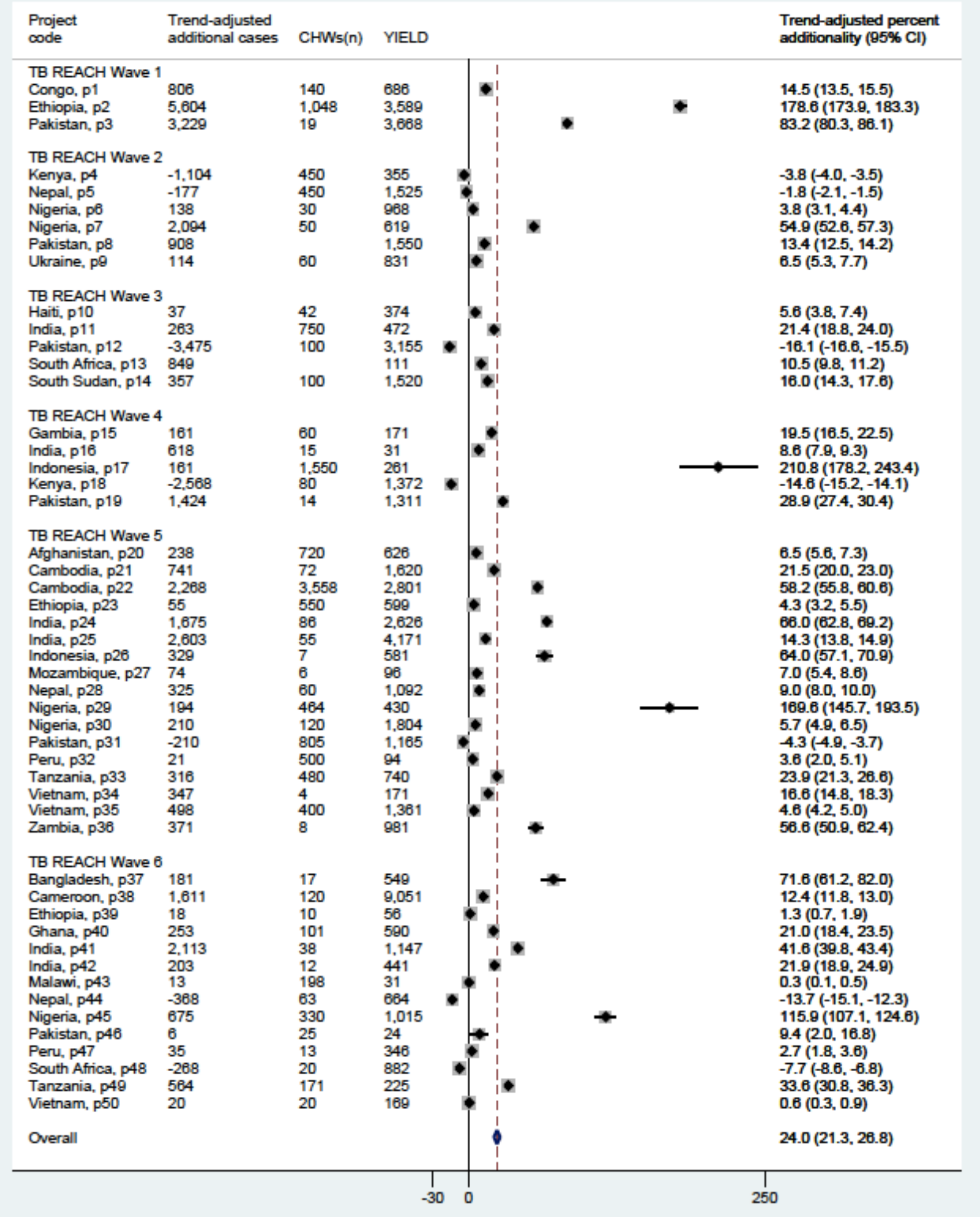

Figure 3

Forest plot of the additionality indicator 
This is a list of supplementary files associated with this preprint. Click to download.

- AdditionalFile1.pdf

- AdditionalFile2.xls 